\title{
WAVELET TRANSFORM OF THE DILATION EQUATION
}

\author{
CARLOS A. CABRELLI and URSULA M. MOLTER ${ }^{1}$
}

(Received 15 November 1993; revised 10 October 1994)

\begin{abstract}
In this article we study the dilation equation $f(x)=\sum_{h} c_{h} f(2 x-h)$ in $\mathscr{L}^{2}(\mathbb{R})$ using a wavelet approach. We see that the structure of Multiresolution Analysis adapts very well to the study of scaling functions. The equation is reduced to an equation in a subspace of $\mathscr{L}^{2}$ (BS) of much lower resolution. This simpler equation is then "wavelet transformed" to obtain a discrete dilation equation. In particular we study the case of compactly supported solutions and we see that conditions for the existence of solutions are given by convergence of infinite products of matrices. These matrices are of the type obtained by Daubechies, and, when the analyzing wavelet is the Haar wavelet, they are exactly the same.
\end{abstract}

\section{Introduction}

In the last years, wavelets have had a strong impact on all the areas of signal processing, particularly in speech, seismic and image processing. An explosion of algorithms which take advantage of their localization properties has appeared in the engineering literature.

One of the advantages of this new tool is the diversity of different wavelet bases that can be constructed. This feature is used, for example, in signal compression with the concept of wavelet packets $[3,4]$, where one can choose from an infinity of different collection of wavelet bases the one that is best adapted to the signal to be coded.

The building block of all these constructions is a solution of a dilation equation of the type $f(t)=\sum_{k} c_{k} f(2 t-k)$. Such a solution, a scaling function, will generate a wavelet basis. For each set of coefficients $c_{k}$, a different wavelet basis with different properties is obtained.

This equation has recently been studied using Fourier analysis methods [8] and direct methods $[10,16]$. In the first case, the periodic function $m(\theta)=\sum_{k} c_{k} e^{i k \theta}$ is used to build the solution. In the second case, the construction of the solution uses

${ }^{1}$ Dept de Matemática, Universidad de Buenos Aires, Pabellón I, 1428 Capital Federal, Argentina.

(C) Australian Mathematical Society, 1996, Serial-fee code 0334-2700/96 
the equation itself. As an example, the "cascade algorithm" obtained by Daubechies allows one to construct compactly supported solutions directly from the coefficients of the dilation equation.

In this paper we adopt a different approach. We fix a Multiresolution Analysis (MA) in $\mathscr{L}^{2}(\mathbb{R})$ with its associated wavelet basis, and we use this rich structure to analyze the dilation equation. The decomposition provided by the MA is very adequate for the study of scaling functions.

When analyzing a function using wavelet methods one can obtain approximations of the function at different levels of resolution. In the particular case that the function under study is a solution of a dilation equation, we observe that higher levels of resolution of the solution can be obtained from lower ones. This allows us to reduce the problem to solve the equation in a much simpler subspace.

This analysis is also carried over to the wavelet transform domain, where we obtain dual relations for the coefficients in the Multiresolution Analysis chosen. Here, the equation is transformed into a discrete dilation equation. This is particularly relevant when we study compactly supported solutions. In this case, the problem is reduced to one of finding an eigenvector of eigenvalue 1 of a matrix whose entries depend on the coefficients of both dilation equations, the one corresponding to the fixed MA and the one under study. The condition for $\mathscr{L}^{\mathbf{2}}$-convergence of the solutions is shown to depend on the convergence of infinite products of two matrices. Similar conditions of convergence have been obtained before by Daubechies [6] and Daubechies and Lagarias [10] using direct methods. It is interesting however, that in the Daubechies case the finite products give the values of an approximation to the solution. In our case, the same products produce the coefficients of the solution, in the wavelet basis, at the level of resolution given by the length of the product.

These products of matrices have now attracted a lot of attention and have occurred in different contexts (see [9], [12], [2], [1], [18], [11], [16]), and revived the concept of Joint Spectral Radius for two matrices introduced much earlier by Rota and Strang in [17].

\section{Wavelets and multiresolution analysis}

The concept of Multiresolution Analysis is due to Mallat and Meyer and creates the framework for the study of wavelets (see [13], [14], [15], [6]). In this section we introduce some notation and state some known results which we will need later. A comprehensive treatment can be found, for example, in the book by Daubechies [7].

Throughout the whole paper, we are going to consider a fixed Multiresolution Analysis (MA) which we will denote $(\varphi, \psi, d)$, where $\varphi, \psi \in \mathscr{L}^{2}(\mathbb{R})$ and $d \in \ell_{2}(\mathbb{Z})$ 
satisfy

$$
\begin{aligned}
\varphi(x) & =\sum_{h} d_{h} \varphi(2 x-h), \\
\psi(x) & =\sum_{h}(-1)^{h} d_{h+1} \varphi(2 x+h), \\
\sum_{k} d_{k} & =2,
\end{aligned}
$$

and the families $\{\varphi(x-k)\}_{k \in \mathbb{Z}}$ and $\{\psi(x-k)\}_{k \in \mathbb{Z}}$ are orthonormal.

If we call

$$
\begin{aligned}
\varphi_{k}^{j}(x) & =2^{j / 2} \varphi\left(2^{j} x-k\right), \\
\psi_{k}^{j}(x) & =2^{j / 2} \psi\left(2^{j} x-k\right) \\
\text { and } \quad V_{j} & =\operatorname{span}\left\{\varphi_{k}^{j}, k \in \mathbb{Z}\right\} \quad j, j \in \mathbb{Z}
\end{aligned}
$$

then the subspaces $V_{j}$ satisfy

$$
\begin{aligned}
\frac{V_{j}}{\bigcup_{k=-\infty}^{+\infty} V_{k}}=V_{j+1}, \\
f \in \mathscr{L}_{j}(\mathbb{R}), \\
f \in \mathbb{V}_{j} \Longleftrightarrow f(2 x) \in V_{j+1},
\end{aligned}
$$

Let $W_{j}=\overline{\operatorname{span}\left\{\psi_{k}^{j}, k \in \mathbb{Z}\right\}}$. Then $W_{j}=V_{j}^{\perp}$ in $V_{j+1}$ and $V_{j+1}=V_{j} \oplus W_{j}$. The space $\mathscr{L}^{2}(\mathbb{R})$ can be decomposed as

$$
\mathscr{L}^{2}(\mathbb{R})=V_{r} \oplus W_{r}^{+} \quad \forall r \in \mathbb{Z},
$$

where

$$
W_{j}^{+}=\overline{\bigoplus_{k \geq j} W_{k}}
$$

Define $P_{j}$ to be the orthogonal projection onto $V_{j}$, and $Q_{j}$ the orthogonal projection onto $W_{j}$. If for each $f \in \mathscr{L}^{2}(\mathbb{R})$ we call $f_{j}=P_{j}(f)$ and $g_{j}=Q_{j}(f)$, then each of the sequences $\left\{f_{j}\right\}$ and $\left\{g_{j}\right\}$ define $f$ uniquely in $\mathscr{L}^{2}$ and we have

$$
f_{j} \stackrel{\mathscr{L}^{2}}{\rightarrow} f \quad \text { and } \quad f_{j}=\sum_{r=-\infty}^{j-1} g_{r} \quad \text { in } \quad \mathscr{L}^{2} .
$$




\section{Dilation equation and multiresolution analysis}

In this section we study the dilation equation using our fixed MA. If $c \in \ell_{2}(\mathbb{Z})$ we want to find solutions (scaling functions) for the equation

$$
f(x)=\sum_{h} c_{h} f(2 x-h)
$$

where

$$
\sum c_{2 k}=1=\sum c_{2 k+1}
$$

This is a usual assumption. Let us therefore look at the interplay between the MA and the dilation equation in "time-domain". For this, if $T: \mathscr{L}^{2}(\mathbb{R}) \longrightarrow \mathscr{L}^{2}(\mathbb{R})$ is the operator

$$
(T f)(x)=\sum_{h} c_{h} f(2 x-h), \quad f \in \mathscr{L}^{2}(\mathbb{R}),
$$

then

$$
\|T\|_{\mathrm{op}} \leq \frac{1}{\sqrt{2}} \sum_{h}\left|c_{h}\right|
$$

Therefore, if $\left\{c_{n}\right\}$ is in $\ell_{1}(\mathbb{Z}), T$ is a bounded linear operator in $\mathscr{L}^{2}(\mathbb{R})$. Note that scaling functions are fixed points of $T$ and the dilation equation becomes $T f=f$. By the properties of an MA we also have that $T V_{j} \subseteq V_{j+1}$ and $T W_{j} \subseteq W_{j+1}$ for $j \geq 0$.

Note that if $f$ is any function in $\mathscr{L}^{2}, P_{\ell}(f)=P_{\ell}\left(f_{j}\right), \ell<j$. Hence if one knows the projection of $f$ onto $V_{j}$, one automatically knows the projection onto any $V_{\ell}$ with $\ell \leq j$.

If in addition $f$ is a solution of the dilation equation, then the next proposition states that the projection at some level of resolution can be obtained from the projection at lower resolution.

PROPOSITION 3.1. If $f$ satisfies the dilation equation(4), then

$$
\begin{aligned}
& f_{j}(x)=\sum_{h} c_{h} f_{j-1}(2 x-h) \text { and } \\
& g_{j}(x)=\sum_{h} c_{h} g_{j-1}(2 x-h)
\end{aligned}
$$

for all $j \geq 1$. 
PROOF. Since $\left\{\varphi_{k}^{\ell}\right\}$ are an orthonormal basis of $V_{\ell}$, we have

$$
f_{j-1}(2 x-h)=\sum_{k}\left(\int f(u) \varphi_{k}^{j-1}(u) d u\right) \varphi_{k}^{j-1}(2 x-h) .
$$

Changing the summation indexes, making an appropriate change of variables, and using

$$
\varphi_{k}^{\ell}(x-h)=\varphi_{2^{\ell} h+k}^{\ell}(x) \quad \text { and } \quad \varphi_{k}^{\ell-1}(x)=\frac{1}{\sqrt{2}} \varphi_{k}^{\ell}\left(\frac{x}{2}\right),
$$

we obtain

$$
\begin{aligned}
f_{j-1}(2 x-h) & =\sum_{s}\left(\int f(u) \varphi_{s-2^{j-1} h}^{j-1}(u) d u\right) \varphi_{s}^{j-1}(2 x) \\
& =\sum_{s}\left(\frac{1}{2} \int f(u) \varphi_{s-2^{j-1} h}^{j}\left(\frac{u}{2}\right) d u\right) \varphi_{s}^{j}(x) \\
& =\sum_{s}\left(\frac{1}{2} \int f(u) \varphi_{s}^{j}\left(\frac{u+h}{2}\right) d u\right) \varphi_{s}^{j}(x) \\
& =\sum_{s}\left(\int f(2 t-h) \varphi_{s}^{j}(t) d t\right) \varphi_{s}^{j}(x)
\end{aligned}
$$

On the other hand, since $f_{j} \in V_{j}$,

$$
f_{j}(x)=\sum_{s}\left(\int f(t) \varphi_{s}^{j}(t) d t\right) \varphi_{s}^{j}(x),
$$

and therefore, if $f$ satisfies the dilation equation $f(x)=\sum_{h} c_{h} f(2 x-h)$, we have

$$
\begin{aligned}
f_{j}(x) & =\sum_{s}\left(\sum_{h} c_{h} \int f(2 t-h) \varphi_{s}^{j}(t) d t\right) \varphi_{s}^{j}(x), \\
& =\sum_{h} c_{h}\left(\sum_{s}\left(\int f(2 t-h) \varphi_{s}^{j}(t) d t\right) \varphi_{s}^{j}(x)\right),
\end{aligned}
$$

which by (7) yields the desired result.

The equation for $g_{j}$ is obtained similarly.

Inductively, formulae (6) yield

$$
\begin{aligned}
& f_{j}(x)=\sum_{h_{1} \ldots h_{j}} c_{h_{1}} \ldots c_{h_{j}} f_{0}\left(2^{j} x-\tau_{j}(h)\right), \\
& g_{j}(x)=\sum_{h_{1} \ldots h_{j}} c_{h_{1}} \ldots c_{h_{j}} g_{0}\left(2^{j} x-\tau_{j}(h)\right),
\end{aligned}
$$

where $\tau_{j}(h)=\sum_{i=1}^{j} 2^{j-i} h_{i}$.

An interesting corollary of Proposition 3.1 is the following result. 
COROLLARY 3.1. If $f \neq 0$ is a scaling function, then $f$ can not be orthogonal to any $V_{j}$ of any $M A$.

The last proposition (as well as its corresponding one in the next section) is the key to our method, since if $f$ is a scaling function, we are able to determine $f$ (by means of finding all its projections) just by finding its projection onto some $V_{j}$.

Thus, by the recursive character of Proposition 3.1, in order to find solutions of (4) it is enough to find the projections of that solution on $V_{0}$ a much smaller space. The next theorem tells us which condition the projection on $V_{0}$, of the solution of (4) has to satisfy.

THEOREM 3.1. If $f$ is an $\mathscr{L}^{2}$ scaling function of (4) then $f_{0}=P_{0} f \in V_{0}$ and $g_{0}=$ $Q_{0} f \in W_{0}$ satisfy

$$
\begin{gathered}
P_{0} T f_{0}=f_{0} \text { and } \\
\sum_{n \geq 0} T^{n} g_{0} \text { converges in } \mathscr{L}^{\mathbf{2}}(\mathbb{R}) .
\end{gathered}
$$

Conversely, if $h \in V_{0}$ satisfies

(i) $h$ is a solution of $\left(P_{0} T-I\right) h=0$, and

(ii) the function $g \equiv Q_{0} T h$ is such that $\sum_{n \geq 0} T^{n} g$ converges in $\mathscr{L}^{2}(\mathbb{R})$, then $f \equiv h+\sum_{n \geq 0} T^{n} g$ is a scaling function of (4).

PROOF. From Proposition 3.1 we know that if $f$ is a scaling function of (4), then $f_{j}=T f_{j-1}$ and $g_{j}=T g_{j-1}$ for $j \geq 1$. If $j=1$, then

$$
T f_{0}=f_{1}=f_{0}+g_{0} \text {. }
$$

This says that $P_{0} T f_{0}=f_{0}$ or equivalently, $\left(P_{0} T-I\right) f_{0}=0$ in $\mathscr{L}^{2}(\mathbb{R})$.

Now since $\mathscr{L}^{2}(\mathbb{R})=V_{0} \oplus \bigoplus_{k \geq 0} W_{k}$, we can write $f$ as $f=f_{0}+\sum_{k \geq 0} g_{k}$ and then by (6), $f=f_{0}+\sum_{k \geq 0} T^{k} g_{0}$ and hence $\sum_{k} T^{k} g_{0}$ converges in $\mathscr{L}^{2}$.

Conversely, if we assume now that $h \in V_{0}$ satisfies (8) and (9) and define $f \equiv$ $h+\sum_{n \geq 0} T^{n} g$, we see that

$$
T f=T h+\sum_{n \geq 0} T^{n+1} g
$$

But

$$
T h=P_{0} T h+Q_{0} T h=h+g .
$$

Thus

$$
T f=h+\sum_{n \geq 0} T^{n} g=f .
$$

Note also that $f=\lim _{n \rightarrow+\infty} T^{n} h\left(\right.$ in $\mathscr{L}^{2}$ ). 
The above theorem shows that in order to solve the dilation equation, it is enough to solve the equation $P_{0} T h=h$ in the subspace $V_{0}$ of some MA, a much simpler subspace. To be more precise, if we define the subspaces

$$
\begin{aligned}
H & =\left\{h \in V_{0}: P_{0} T h=h\right\}, \\
S & =\left\{s \in V_{0}: Q_{0} T s \in U\right\}
\end{aligned}
$$

of $V_{0}$, where $U \subset W_{0}$ is defined by

$$
U=\left\{g \in W_{0}: \sum_{n=1}^{\infty}\left\|T^{n} g\right\|_{2}<+\infty\right\},
$$

then we have that $f$ is a solution of the dilation equation (1) if and only $f$ belongs to $H \cap S$.

Hence, in order to solve the dilation equation, we need to find the intersection of two subspaces of $V_{0}$.

The condition that defines $H$ can be expressed in terms of its coefficients in $V_{0}$, that is,

$$
P_{0} T h=h, \quad h \in V_{0}
$$

is equivalent to

$$
T h-h \perp V_{0} \quad h \in V_{0} \quad \text { or } \quad\left\langle T h-h, \varphi_{k}\right\rangle=0 \quad \forall k .
$$

If we set $h_{k}=\left\langle h, \varphi_{k}\right\rangle$ then from (11) we obtain

$$
\sum_{k} h_{k}\left\langle T \varphi_{k}, \varphi_{j}\right\rangle=h_{j} \quad\left\{h_{k}\right\} \in \ell_{2},
$$

and finally, if we call $R_{k j}=\left\langle T \varphi_{k}, \varphi_{j}\right\rangle k, j \in \mathbb{Z}$, we obtain that

$$
h \in H \quad \text { if and only if } \quad \sum_{k} h_{k} R_{k j}=h_{j} \quad\left\{h_{k}\right\} \in \boldsymbol{\ell}_{\mathbf{2}} .
$$

It is not clear, however, if it is possible to find a good characterization of the elements of $S$, not even in the compactly supported case, which we shall analyze later.

\section{Dilation equation and wavelet transform}

Let us now look at the wavelet-transform domain. Consider the $\operatorname{MA}(\varphi, \psi, d)$, and the coefficients of $f$ in the basis of $V_{j}$ and $W_{j}$ respectively $\left\{a_{k}^{j}\right\}_{k \in \mathbb{Z}}$ and $\left\{b_{k}^{j}\right\}_{k \in \mathbb{Z}}$, that is,

$$
\begin{aligned}
& a_{k}^{j}=\left\langle f, \varphi_{k}^{j}\right\rangle \quad j, k \in \mathbb{Z} \quad \text { and } \\
& b_{k}^{j}=\left\langle f, \psi_{k}^{j}\right\rangle \quad j, k \in \mathbb{Z} .
\end{aligned}
$$


Then

$$
\begin{aligned}
f & =\sum_{k, j \in \mathbb{Z} \times \mathbb{Z}} b_{k}^{j} \psi_{k}^{j}, \\
f_{j} & =P_{j}(f)=\sum_{k \in \mathbb{Z}} a_{k}^{j} \varphi_{k}^{j} \quad \text { in } \mathscr{L}^{2}(\mathbb{R}) \quad \text { and } \\
g_{j} & =Q_{j}(f)=\sum_{k \in \mathbb{Z}} b_{k}^{j} \psi_{k}^{j} .
\end{aligned}
$$

The double sequence $\left\{b_{k}^{j}\right\}$ is sometimes referred to as the wavelet transform or the coefficients of $f$ with respect to the MA $(\varphi, \psi, d)$.

We will now see how the properties of the preceding section are translated into properties of the coefficients of $f$. The following result, due to Mallat, shows how from the coefficients of $f$ in $V_{j+1}$ one can obtain the coefficients of $f$ in any $V_{k}, k \leq j$.

PROPOSITION 4.1 (Mallat). Let $f \in \mathscr{L}^{2}(\mathbb{R})$ and $(\varphi, \psi, d)$ be an MA. For each $j$ in $\mathbb{Z}$, the coefficients of $f$ in $V_{j}$ and $W_{j}$ can be obtained from the coefficients of $f$ in $\vec{V}_{j+1}$ and the coefficients $\left\{d_{k}\right\}$ through the formulae

$$
a_{k}^{j}=\frac{1}{\sqrt{2}} \sum_{h} d_{h} a_{2 k+h}^{j+1} \quad b_{k}^{j}=\frac{1}{\sqrt{2}} \sum_{h}(-1)^{h} d_{h+1} a_{2 k-h}^{j+1} .
$$

Therefore all the coefficients of $f$ in $V_{\ell}$ and $W_{\ell}$ for $\ell \leq j$ can be obtained from the ones in $V_{j+1}$ and the coefficients $\left\{d_{k}\right\}$.

PROOF. Since $\varphi$ is a scaling function of (1), it follows that

$$
\varphi_{k}^{j}=\frac{1}{\sqrt{2}} \sum_{h} d_{h} \varphi_{2 k+h}^{j+1} \quad \text { and } \quad \psi_{k}^{j}=\frac{1}{\sqrt{2}} \sum_{h}(-1)^{h} d_{h+1} \varphi_{2 k-h}^{j+1} .
$$

On calculating the coefficients $\left\{a_{k}^{j}\right\}$ and $\left\{b_{k}^{j}\right\}$ for $f \in \mathscr{L}^{2}(\mathbb{R})$ as in (12) and using (14), we obtain immediately the desired formulae.

In addition, since $V_{j+1}=V_{j} \oplus W_{j}$ we have also that

$$
a_{s}^{j+1}=\frac{1}{\sqrt{2}} \sum_{k} a_{k}^{j} d_{s-2 k}+\frac{1}{\sqrt{2}} \sum_{k} b_{k}^{j}(-1)^{2 k-s} d_{2 k-s+1} \text {. }
$$

To see this note that

$$
a_{s}^{j+1}=\left\langle f, \varphi_{s}^{j+1}\right\rangle=\left\langle f_{j+1}, \varphi_{s}^{j+1}\right\rangle=\left\langle f_{j}+g_{j}, \varphi_{s}^{j+1}\right\rangle .
$$

Using $f_{j}=\sum_{k} a_{k}^{j} \varphi_{k}^{j}$ and $g_{j}=\sum_{k} b_{k}^{j} \psi_{k}^{j}$, together with (14) and the orthogonality of the sets $\varphi_{k}^{j}$ and $\psi_{k}^{j}$, yields the desired result. 
This shows that the coefficients $a_{k}^{j}$ of $f$ in $V_{j+1}$ can be obtained from the coefficients of $f$ in $V_{j}$ and $W_{j}$. In general, no such relation holds for the coefficients $b_{k}^{j}$ of $f$ in $W_{j}$. However, if $f$ is a solution to the dilation equation, we can improve the previous result.

Let $f$ be an arbitrary solution of (4). Our first observation, when we look at the wavelet transform of $f$, is that its coefficients at higher resolution can be obtained from the ones at lower resolution starting at resolution $\left(V_{0}\right)$. In other words, the constraint (4) forces $f$ to satisfy a kind of converse of Proposition 4.1.

PROPOSITION 4.2. If $f \in \mathscr{L}^{2}$ is a solution of the dilation equation

$$
f(x)=\sum_{h} c_{h} f(2 x-h),
$$

then for each $j \geq 1$ the coefficients of $f$ in $V_{j}$ and $W_{j}$ can be obtained from the coefficients of $f$ in $V_{j-1}$ and $W_{j-1}$ and the coefficients $\left\{c_{k}\right\}$ through the formulae

$$
\begin{aligned}
a_{k}^{j} & =\frac{1}{\sqrt{2}} \sum_{h} c_{h} a_{k-2^{j-1} h}^{j-1} \quad \forall k \quad \forall j \geq 1, \\
b_{k}^{j} & =\frac{1}{\sqrt{2}} \sum_{h} c_{h} b_{k-2^{j-1} h}^{j-1} \quad \forall k \forall j \geq 1,
\end{aligned}
$$

and also

$$
\begin{aligned}
& a_{k}^{j}=\left(\frac{1}{\sqrt{2}}\right)^{j} \sum_{h_{1} \ldots h_{j}} c_{h_{1}} \ldots c_{h_{j}} a_{k-\tau_{j}(h)}^{0} \quad \forall k \forall j \geq 1, \\
& b_{k}^{j}=\left(\frac{1}{\sqrt{2}}\right)^{j} \sum_{h_{1} \ldots h_{j}} c_{h_{1}} \ldots c_{h_{j}} b_{k-\tau_{j}(h)}^{0}, \quad \forall k \quad \forall j \geq 1,
\end{aligned}
$$

with $\tau_{s}(x)=\sum_{i=1}^{s} 2^{s-i} x_{i}$.

PROOF. Using the fact that $f$ satisfies the dilation equation and applying several changes of variables in the integrals, we obtain the equalities:

$$
\begin{aligned}
b_{k}^{j} & =\left\langle f, \psi_{k}^{j}\right\rangle=\int f(x) 2^{\frac{j}{2}} \psi\left(2^{j} x-k\right) d x \\
& =\sum_{h} c_{h} \int f(2 x-h) 2^{\frac{j}{2}} \psi\left(2^{j} x-k\right) d x \\
& =\frac{1}{\sqrt{2}} \sum_{h} c_{h} \int f(u) 2^{\frac{j-1}{2}} \psi\left(2^{j-1} u-\left(k-2^{j-1} h\right)\right) d u \\
& =\frac{1}{\sqrt{2}} \sum_{h} c_{h} \int f(u) \psi_{k-2^{j-1} h}^{j-1}(u) d u \\
& =\frac{1}{\sqrt{2}} \sum_{h} c_{h} b_{k-2^{j-1} h}^{j-1} .
\end{aligned}
$$


The equation for $a_{k}^{j}$ is obtained the same way, and recursively one gets (16).

This proposition is the discrete version of Proposition 3.1, since it shows that if $f$ is a solution of the dilation equation, we are able to determine $f-$ that is, to know its wavelet transform - just by knowing its coefficients at some level $V_{j}, j \geq 0$ and then applying (13) and (15) to obtain the coefficients at level $\ell<j$ and $\ell>j$ respectively.

Combining these equations, we have that if $f$ is a solution of the dilation equation (4), then its coefficients in $V_{j}$ and $W_{j}$ for nonnegative $j$ have to satisfy a condition that involves the coefficients $\left\{c_{k}\right\}$ and $\left\{d_{k}\right\}$.

COROLLARY 4.1. If $f$ is a solution of (4), then $\forall j \geq 0$

$$
a_{k}^{j}=\frac{1}{2} \sum_{h, s} d_{s} c_{h} a_{2 k-2 j h+s}^{j} \quad b_{k}^{j}=\frac{1}{2} \sum_{h, s}(-1)^{s} d_{s+1} c_{h} a_{2 k-2^{j} h-s}^{j} .
$$

Taking $j=0$, and setting $a_{k}^{0}=x_{k}$, we get that

$$
x_{k}=\frac{1}{2} \sum_{h, s} d_{s} c_{h} x_{2 k-h+s}
$$

and changing variables in the double sum, we obtain

$$
x_{k}=\frac{1}{2} \sum_{t} r_{t} x_{2 k-t},
$$

where $r_{t}=\sum_{s} d_{s} c_{t+s}$ is the crosscorrelation between the coefficients of the two dilation equations. This is the equivalent to condition (8) in the transform domain.

Equation (19) represents a discrete dilation equation in $\ell_{2}(\mathbb{Z})$ associated with the wavelet transform $\left(d_{k}\right)$. To be precise, (19) represents the wavelet transform of (4). We call any solution of this equation a scaling sequence.

Define $H, G: \ell_{2}(\mathbb{Z}) \rightarrow \ell_{2}(\mathbb{Z})$ by

$$
\begin{aligned}
& (H x)_{k}=\frac{1}{2} \sum_{h, s} d_{s} c_{h} x_{2 k-h+s}, \\
& (G x)_{k}=\frac{1}{2} \sum_{h, s}(-1)^{s} d_{s+1} c_{h} x_{2 k-h-s} \quad \forall x \in \ell_{\mathbf{2}}(\mathbb{Z}) .
\end{aligned}
$$

We then have that $x \in \ell_{2}(\mathbb{Z})$ is a scaling sequence if and only if $H x=x$. That is, $x$ is an eigensequence of eigenvalue 1 .

The next result is the transform domain version of Theorem 3.1.

THEOREM 4.1. If $f$ is a $\mathscr{L}^{2}$-solution of (4) then $\left\{a_{k}^{0}(f)\right\}_{k}$ is a scaling sequence of (19) and $\left\{b_{k}^{j}(f)\right\}_{j, k}, j \geq 0$ is an $\ell_{2}$ sequence. Conversely, if $x^{0} \in \ell_{2}(\mathbb{Z})$ satisfies 
(i) $H x^{0}=x^{0}$, that is $x^{0}$ is a scaling sequence of (19), and

(ii) the sequence $y^{0}=G x^{0}$ is such that $\sum_{j \geq 0} \sum_{k}\left|y_{k}^{j}\right|^{2}<+\infty$ (where $y_{k}^{j}=\frac{1}{\sqrt{2}} \sum_{h} c_{h} y_{k-2^{j-1} h}^{j-1} \quad j \geq 1, k \in \mathbb{Z}$ ).

then $f \equiv \sum_{k} x_{k} \varphi_{k}+\sum_{j \geq 0} \sum_{k} y_{k}^{j} \psi_{k}^{j}$ is an $\mathscr{L}^{2}$-solution of (4),

PROOF. By the previous remarks, if $f$ is a $\mathscr{L}^{2}$-solution of $(4)$ then $a^{0}(f)$ is a scaling sequence of (19).

For the converse we appeal to Theorem 3.1. Assume $x^{0}$ satisfies (22) and (23) and let $y^{0}=G x^{0}$. Define the functions $h \in V_{0}$ and $g \in W_{0}$ by

$$
h(t)=\sum_{k} x_{k}^{0} \varphi(t-k), \quad g(t)=\sum_{k} y_{k}^{0} \psi(t-k) .
$$

Then on one side

$$
\begin{aligned}
\left\langle T h, \varphi_{k}^{0}\right\rangle & =\sum_{\ell} c_{\ell}\langle h(2 t-\ell), \varphi(t-k)\rangle \\
& =\sum_{\ell, s} c_{\ell} x_{s}^{0}\langle\varphi(2 t-\ell-s), \varphi(t-k)\rangle,
\end{aligned}
$$

and since $\varphi$ satisfies the dilation equation (1) and by orthonormality of $\left\{\varphi_{k}^{j}\right\}$, we obtain

$$
\begin{aligned}
\left\langle T h, \varphi_{k}^{0}\right\rangle & =\sum_{\ell, s, r} c_{\ell} d_{r} x_{s}^{0}\langle\varphi(2 t-\ell-s), \varphi(2 t-2 k-r)\rangle \\
& =\sum_{\ell, s, r} c_{\ell} d_{r} x_{s}^{0} \frac{1}{2} \delta_{l+s, 2 k+r} \\
& =\frac{1}{2} \sum_{\ell, r} c_{\ell} d_{r} x_{2 k+r-\ell}^{0}=x_{k}^{0}=\left\langle h, \varphi_{k}^{0}\right\rangle .
\end{aligned}
$$

Thus $T h$ and $h$ share the same coefficients on $V_{0}$, that is, $P_{0} T h=h$.

On the other hand

$$
\left\langle T h, \psi_{k}^{0}\right\rangle=\sum_{\ell, s} c_{\ell} x_{s}^{0}\langle\varphi(2 t-\ell-s), \psi(t-k)\rangle,
$$

and now, by the orthonormality of $\left\{\psi_{k}^{j}\right\}$ and (2),

$$
\begin{aligned}
\left\langle T h, \psi_{k}^{0}\right\rangle & =\sum_{\ell, s, r}(-1)^{r} c_{\ell} d_{r+1} x_{s}^{0}\langle\varphi(2 t-\ell-s), \varphi(2 t-2 k+r)\rangle \\
& =\frac{1}{2} \sum_{\ell, r}(-1)^{r} c_{\ell} d_{r+1} x_{2 k-r-\ell}^{0}=\left(G x^{0}\right)_{k}=y_{k}^{0}=\left\langle g, \psi_{k}^{0}\right\rangle .
\end{aligned}
$$

Hence $Q_{0} T h=g$. 
Since $g \in W_{0}$, we have $T^{j} g \in W_{j}$ with coefficients $y_{k}^{j}$. To see this we calculate them inductively.

$$
\begin{aligned}
\left\langle T^{j} g, \psi_{k}^{j}\right\rangle & =\sum_{\ell} c_{\ell}\left\langle\left(T^{j-1} g\right)(2 t-\ell), \psi_{k}^{j}(t)\right\rangle \\
& =\sum_{\ell, s} c_{\ell} y_{s}^{j-1}\left\langle\psi_{s}^{j-1}(2 t-\ell), \psi_{k}^{j}(t)\right\rangle \\
& =\frac{1}{\sqrt{2}} \sum_{\ell, s} c_{\ell} y_{s}^{j-1}\left\langle\psi_{2^{j-1} \ell+s}^{j}, \psi_{k}^{j}\right\rangle \\
& =\frac{1}{\sqrt{2}} \sum_{\ell} c_{\ell} y_{k-2^{j-1} \ell}^{j-1}=y_{k}^{j} .
\end{aligned}
$$

This property shows, in particular that $T^{\ell} g \perp T^{s} g$ for $\ell \neq s$ and therefore that

$$
\left\|\sum_{j \geq 0} T^{j} g\right\|^{2}=\sum_{j \geq 0}\left\|T^{j} g\right\|^{2}=\sum_{j \geq 0} \sum_{k}\left|y_{k}^{j}\right|<+\infty .
$$

We therefore have proved that the function $h$ satisfies (8) and (9) of Theorem 3.1. Then

$$
f=h+\sum_{j \geq 0} T^{j} g=\sum_{j \geq 0} \sum_{k} y_{k}^{j} \psi_{k}^{j}+\sum_{k} x_{k} \varphi_{k}
$$

is an $\mathscr{L}^{2}$-solution of (4).

Therefore, the $\ell_{2}$-solutions of (19) give all the $\mathscr{L}^{2}$ scaling functions. Notice also that the coefficients of the discrete dilation equation depend on the Multiresolution Analysis used. The role of the coefficients $\left(d_{s}\right)$ in (19) can be seen from (18), which gives

$$
x_{k}=\sum_{h} c_{h}\left[\sum_{s} \frac{d_{s}}{2} x_{(2 k-h)+s}\right] .
$$

Hence, since $\sum d_{s}=2$, the expression in brackets represents a weight-average of the consecutive values of $x$.

4.1. Compactly supported solutions Let us analyze the case of compactly supported solutions. We assume now that our function $\varphi$ of the analyzing MA is also compactly supported. Let the coefficients of the dilation equations $\left\{c_{k}\right\},\left\{d_{k}\right\}$ be finite sequences, that is, $c=c_{0}, \ldots, c_{N}$, and $d=d_{0}, \ldots, d_{M}$, with $N$ and $M$ odd positive integers.

These assumptions imply that $\operatorname{supp}(\varphi) \subseteq[0, M]$ and that if $f$ is a compactly supported scaling function, $\operatorname{supp}(f) \subseteq[0, N]$. Furthermore, the coefficients $\left\{a_{k}^{0}(f)\right\}$ 
of $f$ in the basis $\left\{\varphi_{k}^{0}\right\}$ of $V_{0}$ are zero for $k \notin\{-(M-1), \ldots, N-1\}$, and the coefficients $\left\{b_{k}^{0}(f)\right\}$ of $f$ in the basis $\left\{\psi_{k}^{0}\right\}$ of $W_{0}$ are zero for $k \notin\left\{-\frac{M-1}{2}, \ldots, N+\frac{M-1}{2}-1\right\}$. (This is straightforward as $f$ has compact support, satisfies (4) with a finite sequence $c_{k}$ and the coefficients in $V_{0}$ satisfy (19).) This says that, when looking for compactly supported solutions, we only have to find one set of $M+N-1$ coefficients. That is, by Theorem 4.1 we need to solve

$$
x_{k}=\frac{1}{2} \sum_{s} d_{s} \sum_{l} c_{2 k-l+s} x_{l} \quad \text { for } \quad k \in\{-(M-1), \ldots, N-1\}
$$

and then define

$y_{k}=\frac{1}{2} \sum_{s}(-1)^{s} d_{s+1} \sum_{\ell} c_{2 k-\ell-s} x_{\ell} \quad$ for $\quad k \in\left\{-\frac{M-1}{2}, \ldots, N+\frac{M-1}{2}-1\right\}$.

Define now $L=N+M-1, \alpha=\left(\alpha_{0}, \ldots, \alpha_{L-1}\right)$ and $\beta=\left(\beta_{0}, \ldots, \beta_{L-1}\right)$ by

$$
\boldsymbol{\alpha}_{k}=x_{k-(M-1)} \quad \text { and } \quad \beta_{k}=y_{k-\frac{M-1}{2}} .
$$

Now (24) and (4.1) can be written in matrix form. For this define the $L \times L$ matrices $A_{s}$ for $s=0, \ldots, M$ with entries

$$
a_{i j}^{s}=c_{2 i-j-M+s} \quad 1 \leq i, j \leq L
$$

If we call

$$
H=\frac{1}{2} \sum_{s} d_{s} A_{s}, \quad G=\frac{1}{2} \sum_{s}(-1)^{s} d_{s+1} A_{1-s},
$$

then (24) and (4.1) become $H \alpha=\alpha$ and $\beta=G \alpha$. Notice that the entries of the matrix $H$ can be written as $h_{i j}=\frac{1}{2} r_{2 i-j-M}$ where $r_{t}=\sum_{s} d_{s} c_{i+s}$ is the crosscorrelation between the coefficients of the two dilation equations.

By (5), the columns of $A_{s}$ for $s=0, \ldots, M$ add up to 1 . Therefore the vector $(1,1, \ldots, 1)$ is an eigenvector of eigenvalue 1 of $H^{t}$, which implies that (24) always has a solution.

In the Haar case $\left(M=1, d_{0}=d_{1}=1\right)$ the matrices $A_{0}$ and $A_{1}$ turn out to be the matrices obtained by Daubechies in [10], and

$$
H=\frac{1}{2}\left(A_{0}+A_{1}\right) \quad \text { and } \quad G=\frac{1}{2}\left(A_{1}-A_{0}\right) .
$$

So far we have obtained that in order to solve (4) we need to solve $H \alpha=\alpha, \alpha \in \mathbb{R}^{L}$. The vector $\alpha$ will represent the coefficients in the basis of $V_{0}$ of the projection of the solution on $V_{0}$ and $\beta=G \alpha$ the coefficients in the basis of $W_{0}$ of the projection of the solution on $W_{0}$. 
In order to find the coefficients of the projection of the solution in $W_{j}, j \geq 1$, we use (15), that is, we define inductively

$$
\begin{aligned}
\alpha_{k}^{j} & =\frac{1}{\sqrt{2}} \sum_{h} c_{h} \alpha_{k-2^{j-1} h}^{j-1} & \forall k & \forall j \geq 1, \\
\beta_{k}^{j} & =\frac{1}{\sqrt{2}} \sum_{h} c_{h} \beta_{k-2^{j-1} h}^{j-1} & \forall k & \forall j \geq 1,
\end{aligned}
$$

Since we start with a vector with $L$ components at level 0 , at level $j$ we will have $2^{j} L$ coefficients. Note that applying (27) doubles the number of coefficients at each step. Let us call

$$
\alpha^{j}=\left(\alpha_{0}^{j}, \ldots, \alpha_{2^{j} L-1}^{j}\right), \quad \beta^{j}=\left(\beta_{0}^{j}, \ldots, \beta_{2^{j} L-1}^{j}\right) .
$$

Observe that due to (26) we have the relations $\alpha_{k}^{j}=x_{k-(M-1)}^{j}, \beta_{k}^{j}=y_{k-\frac{M-1}{2}}^{j}$ at each level $j \geq 0$.

We see now that (27) admits a matrix form. For a fixed $j \geq 0$ let us split the $2^{j} L$ coefficients $\beta_{0}^{j}, \ldots \beta_{2^{j} L-1}^{j}$ in the following way.

Let $k$ be an integer, $k \in\left\{0,1, \ldots, 2^{j}-1\right\}$. If we collect in a vector $w_{j}(k)$ the $L$ coefficients

$$
w_{j}(k)=\left(\beta_{k}^{j}, \beta_{k+2^{j}}^{j}, \ldots, \beta_{k+(L-1) 2^{j}}^{j}\right)
$$

that have indices with remainder $k\left(\bmod 2^{j}\right)$, then the function $w_{j}:\left\{0, \ldots, 2^{j}-1\right\} \rightarrow \mathbb{R}^{L}$ incorporates all the information at level $j$. Note that $w_{0}$ is only defined at $\{0\}$ and $w_{0}(0)=\beta$. We can see from (27) that for $k \in\left\{0, \ldots, 2^{j+1}-1\right\}$,

$$
w_{j+1}(k)= \begin{cases}B_{0} w_{j}(k) & k \in\left\{0, \ldots, 2^{j}-1\right\} \\ B_{1} w_{j}\left(k-2^{j}\right) & k \in\left\{2^{j}, \ldots, 2^{j+1}-1\right\},\end{cases}
$$

where $B_{0}=A_{M-1} / \sqrt{2}$ and $B_{1}=A_{M} / \sqrt{2}$.

If $k \in\left\{0, \ldots, 2^{j+1}-1\right\}$ and its binary expansion is $k=\sum_{t=0}^{j} k_{t} 2^{t}, k_{t} \in\{0,1\}$, then

$$
w_{j+1}(k)=B_{k_{0}} B_{k_{1}} \cdots B_{k_{j}} w_{0}
$$

That is, the $2^{j+1} L$ coefficients at level $j+1$ are all the products of length $j+1$ applied to $w_{0}=\beta$.

It is interesting to notice that the entries of the matrices $A_{s}$ depend only on the coefficients $\left\{c_{k}\right\}$ of the dilation equation and not on the MA chosen. Hence the coefficients $\left\{\beta_{k}^{j}\right\}$ depend on $\left\{d_{k}\right\}$ only through $\beta=G \alpha$.

These products of matrices have been obtained by Daubechies [6], [10] in the study of the dilation equation and also by Micchelli and Prautzsch [16] in the framework of 
subdivision schemes. In the analysis of the convergence of these products the concept of joint spectral radius for two matrices, introduced by Rota and Strang in [17] plays an important role. See [9], [1], [18], [12], [11], [5] for results in that direction.

If we start the iteration described above with $\alpha$ rather than with $\beta$, then at each step we obtain the coefficients $\left\{\alpha_{k}^{j}\right\}$ of the projection of the scaling function in $V_{j}$, that is we define

$$
v_{0}=\alpha \quad \text { and } \quad v_{j+1}(k)=B_{k_{0}} B_{k_{1}} \cdots B_{k_{j}} v_{0}
$$

with $k=\sum_{i=0}^{j} k_{t} 2^{t}, k_{t} \in\{0,1\}$.

We want to point out here that the matrices $A_{M-1}$ and $A_{M}$ involved in the computation of the coefficients have the last $\frac{M-1}{2}$ rows equal to zero. As a consequence it is enough to consider only $M-1+2^{j} N$ coefficients at level $j$, that is,

$$
\begin{aligned}
& \text { for } V_{j}, \quad x_{-(M-1)}^{j}, \ldots, x_{2^{j}-1}^{j}, \quad \text { and } \\
& \text { for } W_{j}, \quad y_{-\frac{M-1}{2}}^{j}, \ldots, y_{2^{j} N+\frac{M-1}{2}-1}^{j} .
\end{aligned}
$$

In Daubechies' work, the products of length $j$ of the matrices give the values of an approximation of the scaling function when applied to an appropriate vector. Here the same products when applied to a different vector yield the coefficients of the scaling function at level $j$ for some chosen MA. In the Haar case both interpretations in some sense agree, since in that case we have that

$$
f_{j}(x)=2^{\frac{i}{2}} a_{[2 j x]}^{j}(f),
$$

where $[x]$ denotes the integer part of $x$.

\section{Acknowledgments}

Part of this research was carried out while the authors held visiting positions at the Department of Pure Mathematics at the University of Waterloo, Waterloo, Ontario N2L 3G1, Canada.

We wish to acknowledge support from the Faculty of Mathematics (Dean's Office) and the Department of Pure Mathematics of the University of Waterloo. We also want to thank Norm Corbett who carefully read the manuscript and improved its clarity.

\section{References}

[1] M. A. Berger and Y. Wang, "Bounded semigroups of matrices", Linear Alg. Appl. 166 (1992) 21-27.

[2] M. A. Berger and Y. Wang, "Multi-scale dilation equations and iterated function systems", to appear. 
[3] R. Coifman, Y. Meyer and M. V. Wickerhauser, "Wavelet analysis and signal processing", in Wavelets and Their Applications (ed. M. B. Ruskai et. al), (Jones and Bartlett, Boston, 1992) 153-178.

[4] R. Coifman, Y. Meyer and M. V. Wickerhauser, "Size properties of wavelet packets", in Wavelets and Their Applications (ed. M. B. Ruskai et. al), (Jones and Bartlett, Boston, 1992) 453-470.

[5] D. Colella and C. Heil, "Characterizations of scaling functions, I. Continuous solutions", SIAM J. Matrix Anal. Appl. 15 (1994) 496-518.

[6] I. Daubechies, "Orthonormal bases of compactly supported wavelets", Comm. Pure Appl. Math. 41 (1988) 909-996.

[7] I. Daubechies, "Ten lectures on wavelets", CBMS-NSF Series in Applied Mathematics 61 (SIAM Publications, Philadelphia, 1992).

[8] I. Daubechies and J. C. Lagarias, "Two-scale difference equations I. Existence and global regularity of solutions”, SIAM J. Math. Anal. 22 (1991) 1388-1410.

[9] I. Daubechies and J. C. Lagarias, "Sets of matrices all infinite products of which converge", Linear Alg. Appl. 161 (1992) 227-263.

[10] I. Daubechies and J. C. Lagarias, "Two-scale difference equations II. Infinite products of matrices and fractals", SIAM J. Math. Anal. 23 (1992) 1031-1079.

[11] C. Heil and G. Strang, "Continuity of the joint spectral radius", preprint, 1993.

[12] J. C. Lagarias and Y. Wang, "The finitness conjecture for the generalized spectral radius of a set of matrices", preprint, 1992.

[13] S. Mallat, "Multiresolution approximations and wavelet orthonormal basis of $\mathrm{L}^{2}(R)$ ", Trans. Amer. Math. Soc. 315 (1989) 69-87.

[14] I. Meyer, Ondelettes, fonctions splines et analyses graduées (Lectures given at the University of Torino, Italy, 1986).

[15] I. Meyer, Ondelettes et operateurs I (Hermann, 1988).

[16] C. A. Micchelli and H. Prautzsch, "Uniform refinement of curves", Linear Alg. Appl. 114/115 (1989) 841-870.

[17] G. C. Rota and G. Strang, "A note on the joint spectral radius", Indagationes Mathematicae 22 (1960) 379-381.

[18] Y. Wang, "On two-scale dilation equations", to appear. 